\title{
High flow conditioned oxygen therapy for prevention of reintubation in critically ill patients at high risk for extubation failure: a multicenter randomised controlled trial
}

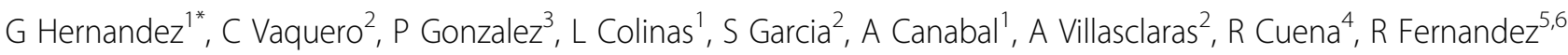 \\ From ESICM LIVES 2015 \\ Berlin, Germany. 3-7 October 2015
}

\section{Introduction}

Critically ill mechanically ventilated patients are usually stratified in two levels according to the risk for reintubation. In low-risk patients high flow conditioned oxygen therapy (HFO) may reduce reintubation, whereas in high-risk patients non-invasive mechanical ventilation (NIMV) to prevent reintubation is gaining acceptance. However, data on the efficacy or safety of HFO in highrisk patients are lacking.

\section{Objectives}

We hypothesized that HFO is an appropriate alternative to NIMV in preventing reintubation in high risk patients. The primary outcome was all-causes and respiratory-related reintubation rate within 72 hours.

\section{Methods}

In this multicenter, randomized, noninferiority trial, we recruited 603 critically ill patients at high-risk for reintubation to be treated after extubation with either HFO ( $\mathrm{n}=$ 290) or NIMV ( $\mathrm{n}=313)$. High-risk for reintubation was defined as the presence of at least any of the following criteria: $>65$ years, cardiac failure, moderate-to-severe COPD, APACHE II $>12$ points at extubation day, body mass index $>30$, airway patency problems, inadequate secretions management, not simple weaning, $\geq 2$ comorbidities, and prolonged mechanical ventilation. NIMV as a rescue therapy was not allowed in the HFO group. Noninferiority was determined as $\mathrm{a}<6 \%$ absolute difference in the risk of the primary outcome.

\section{Results}

Respiratory-related reintubations occurred in 49 (16.9\%) HFO patients and in $50(16 \%)$ NIMV patients (risk difference $0.9 \%$; upper limit of the unilateral 95\%CI 5.9). All-causes reintubation occurred in 67 (23.1\%) HFO patients and in $60(19.2 \%)$ NIMV patients (risk difference 3.9\%; upper limit of the unilateral 95\%CI 9.4\%).

\section{Conclusions}

In critically ill patients at high risk, HFO was noninferior to NIMV in preventing respiratory-related reintubation, but not all-causes reintubation. The study was registered at ClinicalTrials.gov (Identifier: NCT01191489).

\section{Authors' details}

${ }^{1}$ Complejo Hospitalario de Toledo, Intensive Care Medicine, Toledo, Spain. ${ }^{2}$ Hospital Universitario Ramon y Cajal, Intensive Care Medicine, Madrid, Spain. ${ }^{3}$ Hospital Universitario Infanta Sofia, Intensive Care Medicine, San Sebastian de los Reyes, Spain. ${ }^{4}$ Medical Council, Research Unit, Toledo, Spain. ${ }^{5}$ Hospital Sant Joan de Deu-Fundació Althaia, Intensive Care Medicine, Manresa, Spain. ${ }^{6}$ Universitat Internacional de Catalunya. CIBERES, Manresa, Spain.

Published: 1 October 2015

\section{Reference}

1. Maggiore SM, Idone FA, Vaschetto $R$, et al: Nasalhigh-flow versus venture mask oxygen therapy after extubation. Effects on oxygenation, comfort, and clinical outcome. Am J Respir Crit Care Med 2014, 190:282-288.

doi:10.1186/2197-425X-3-S1-A823

Cite this article as: Hernandez et al: High flow conditioned oxygen therapy for prevention of reintubation in critically ill patients at high risk for extubation failure: a multicenter randomised controlled trial. Intensive Care Medicine Experimental 2015 3(Suppl 1):A823. 